Egyptian Journal of Aquatic Biology \& Fisheries

Zoology Department, Faculty of Science,

Ain Shams University, Cairo, Egypt.

ISSN $1110-6131$

Vol. 23(3): $561-573$ (2019)

www.ejabf.journals.ekb.eg

\title{
Effectiveness evaluation of Chrysomya albiceps (Diptera: Calliphoridae) and Musca domestica (Diptera: Muscidae) maggots extracts as antimicrobial and antiviral agent
}

\author{
Moneer S. Amer, Kotb M. Hammad, Ahmed I. Hasaballah*, \\ Ahmed, Z.I. Shehata and Saeed M. Saeed \\ Zoology Department, Faculty of Science, Al-Azhar University, Cairo, Egypt. \\ *Corresponding Author: ahscience09@azhar.edu.eg
}

\section{ARTICLE INFO \\ Article History: \\ Received: Aug. 17, 2019 \\ Accepted:Sept. 15, 2019 \\ Online: Sept. 18, 2019}

Keywords:
Chrysomya albiceps
Musca domestica
Bacteria
HSV
Maggots

\begin{abstract}
The present study aimed to evaluate the antimicrobial activity of Chrysomya albiceps and Musca domestica maggots' extracts produced from different solvents against some bacterial and fungal strains, beside the role of these extracts as anti-HSV therapeutic agents. Results obtained evoked a variable activity against both Gram-positive and Gram-negative bacteria depending on tested species and the solvent used in extraction. The highest antibacterial activity was attained by petroleum ether extract $24 \mathrm{~h}$ post treatment for both Gram-positive and Gram-negative bacteria either by Microbial Growth Inhibition method or by Minimum Inhibitory Concentration method followed by hexane, ethyl acetate and acetone extract. Gram-positive bacterial strains were more sensitive than Gramnegative bacterial strains. Regarding the antifungal activity, tested extracts showed variable antifungal activity. In addition, petroleum ether extracts of tested species exhibited a vital role as potential anti-HSV agent due to their promising antiviral activity. In general, tested extracts induced remarkable effects on both antimicrobial and antiviral activities.
\end{abstract}

\section{INTRODUCTION}

The field of natural product discovery has undergone a tremendous development over the past few decades due to the consequence of several new and revolutionizing drug discovery and development techniques (Fouda et al., 2013; Hassan et al.,2013; Hasaballah, 2015; Wohlleben et al.,2016). Insects are the largest group of the still existing organisms, their individual number account for as much as $80 \%$ of all known fauna and considered a large, unexplored and unexploited source of potentially useful compounds for modern medicine due to their mode of action of non-selective interaction with microbes' cell surface membranes (Leem et al.,1999; Pemberton, 1999; Hancock and Rozek, 2002; Zasloff, 2002; Boman, 2003; Bulet et al.,2004).

A great part of efforts have been achieved for the investigation and reexamination of insect sources to obtain compounds that may possess pharmacological activities. Many authors believe that, it is increasingly possible to use insect extracts as antimicrobial agents to ascertain phylogenetic patterns among insect species, for example (Esser et al., 1979; Wachinger et al.,1998; Fenard et al., 2001; Hou et al., 
2007; Slocinska et al.,2008). In the last few decades, five major groups of proteins act as antimicrobial agents have been isolated from different species of insects: cecropins, insect defensins, attacin-like (glycine-rich) proteins, proline-rich peptides and lysozymes (Hultmark, 1993; Cociancich et al., 1994). Drosomycin, metchinikowin, cecropin $\mathrm{A} \& \mathrm{~B}$ and heliomicin as antifungal peptides/polypeptides isolated from insects (Fehlbaum et al., 1994; Levashina et al., 1995; Lamberty et al., 1999).

Antimicrobial peptides/polypeptides are mainly synthesized in the fat body and released into the hemolymph where they play the crucial role in innate immune system and host defence mechanisms, with a broad-spectrum activity against both Gram-positive and Gram-negative bacteria and fungi (Hoffmann 1995; Hoffmann et al.1996; Januszanis et al.2012).

This study aimed to investigate the possible antimicrobial activity of Chrysomya albiceps (Diptera: Calliphoridae) and Musca domestica (Diptera: Muscidae) maggots petroleum ether, hexane, acetone and ethyl acetate extracts. In addition, to study the potential role of these extracts as anti-HSV therapeutic agents.

\section{MATERIALS AND METHODS}

\section{Colonization of tested flies}

\section{The blowfly, Chrysomya albiceps}

Chrysomya albiceps maggots were collected and transferred to Medical Entomology Insectary, Biology Department, Faculty of Science, Jazan University (KSA) and maintained for several generations under controlled conditions, at temperature of $\left(27 \pm 2^{\circ} \mathrm{C}\right)$, relative humidity $(60 \pm 10 \%)$ and photoperiods (12h light: $12 \mathrm{~h}$ dark). Adults were reared in mesh cages $(30 \times 30 \times 30 \mathrm{~cm})$ with three sides of wire, maggots were feed on an artificial diet (liver), and the emerged flies were feed on milk powder and sucrose solution.

\section{The house fly, Musca domestica}

The housefly maggots were collected by hand trap and maintained for several generations under controlled conditions of temperature $\left(27 \pm 2^{\circ} \mathrm{C}\right)$, relative humidity $(70 \pm 5 \%)$ and photoperiods (12h light: $12 \mathrm{~h}$ dark). The emerged flies were fed on dry diet (milk powder) and sucrose solution (cotton pads soaked in $10 \%$ sucrose solution). Deposited eggs were collected from paper strips or cotton pads of feeding. Larvae were reared on an artificial diet (wheat bran, milk, powder yeast; 200:100:5gm) per $200 \mathrm{ml}$ distilled water according the method described by Busvine, (1962).

\section{Preparation of maggots' extracts}

The extraction was performed according to the methods of Ahn et al.(2000) and Meylears et al.(2002). The extraction was carried out using petroleum ether, hexane, acetone and ethyl acetate solvents.

\section{Antimicrobial bioassay}

\section{Antibacterial activity of tested extracts}

Six pathogenic bacterial strains were used for the antibacterial assay; Staphylococcus aureus (ATCC25923), Staphylococcus pyogenes (ATCC12344) and Bacillus subtilis (ATCC6051) as Gram-positive bacterial strains; whereas, Escherichia coli (ATCC25922DQ), Klebsiella pneumoniae (ATCC11296) and Pseudomonas aeruginosa (ATCC10145) were used as Gram-negative bacterial strains. Microbial growth inhibition was tested using agar well diffusion method (Valgas et al.,2007; Hasaballah \& Elnaggar, 2017). Also, Minimum Inhibitory 
Concentration (MIC) was determined based on the microdilution method by broth microdilution method using 96-well micro-plates (Irith et al.2008).

\section{Antifungal activity of tested extracts}

The fungi Aspergillus flavus, Aspergillus fumigatus, Candida albicans, Geotricum candidum and Penicillium sp. strains were used. All tested microorganisms were supplied by the Microbiology Department, Faculty of Science, Jazan University, KSA. Sucrose-Nitrate agar medium gm/L consisted of: Sucrose, $\mathrm{NaNO}_{3}, \mathrm{~K}_{2} \mathrm{HPO}_{4}, 1.0 \mathrm{mgSO}, 7 \mathrm{H}_{2} \mathrm{O}$ and distilled water, $1000 \mathrm{ml}$ was used in this test. The $\mathrm{pH}$ value was adjusted to 7-7.3 before sterilization (Tadashi, 1975). The detection of inhibitory clear zone around the paper disks is an indication of the antagonistic properties of the extracts under evaluation.

\section{Antiviral assay}

\section{Determination of samples cytotoxicity on VERO cell}

Petroleum ether extract was used to determine the anti-HSV activity. Growth medium was decanted from 96 well micro titer plates after confluent sheet of VERO cell formed. Plate was incubated at $37^{\circ} \mathrm{C}$ and examined frequently for up to 2 days. Cells were checked for any physical signs of toxicity. A $20 \mu \mathrm{l}$ of MTT solution was added to each well. Incubation for 1-5h was done to allow the MTT metabolism. The media dumped off. Plate dried on paper towels to remove residue. Formazan (MTT metabolic product) re-suspend in $200 \mu 1$ DMSO, Placed on a shaking table, $150 \mathrm{rpm}$ for 5 minutes, to thoroughly mix formazan into the solvent. The optical density was read at $560 \mathrm{~nm}$ and subtracts background at $620 \mathrm{~nm}$. The maximum non-toxic concentration of each extract was determined and was used for further biological studies.

\section{Antiviral assay (MTT Assay Protocol)}

Equal volume $(1: 1 \mathrm{v} / \mathrm{v})$ of non-lethal dilution of tested extracts was incubated and the virus suspended for $1 \mathrm{~h}$. A $100 \mu \mathrm{l}$ from viral/ sample suspension was added, placed on a shaking table, 150 rpm for 5 minutes. Incubation at $\left(37^{\circ} \mathrm{C} \& 5 \% \mathrm{CO}_{2}\right)$ was done for 1day to allow the virus to take effect. A 2ml of MTT solution per 96 well plates was prepared at $5 \mathrm{mg} / \mathrm{ml}$ in PBS. A $20 \mu 1$ MTT solution was added to each well, placed on a shaking table, 150rpm for 5 minutes, to thoroughly mix the MTT into the media. The media dumped off. Plate dried on paper towels to remove residue. Formazan (MTT metabolic product) re-suspend in $200 \mu 1$ DMSO, placed on a shaking table, 150rpm for 5 minutes, to thoroughly mix Formazan into the solvent. The optical density was read at 560nm and subtracts background at $620 \mathrm{~nm}$. Optical density should be directly correlated with cell quantity.

\section{Statistical analysis}

The statistical analysis of the data obtained was done according to Armitage, (1974) and Lentner et al.(1982) and the analysis was revised by Quattro-pro for windows program version 2.0 Microsoft, windows version 7.0, graphics were drawn using Harvard Graphics program version 4.0. The obtained data were assessed by calculation of mean (M), standard deviation (SD) and student t-test.

\section{RESULTS}

The blowfly, Chrysomya albiceps

Antimicrobial activity using well diffusion method Antibacterial activity 
Data given in table (1) and figures (1A-1C) represent the effect of petroleum ether, hexane, acetone and ethyl acetate of $C$. albiceps maggots' whole body extracts against different Gram-positive bacterial strains.

Table 1: Antibacterial activity (indicated by growth-inhibition zone) of Chrysomya albiceps maggots' extracts against different strains of Gram-positive bacteria.

\begin{tabular}{ccccccc}
\hline \multirow{2}{*}{ Bacteria } & Gram & \multicolumn{3}{c}{ Growth-inhibition zone in mm caused by extracts } & Standard \\
\cline { 3 - 6 } & $+/-$ & Petroleum ether & Hexane & Acetone & Ethyl acetate & (Ampicillin) \\
\hline Staphylococcus aureus & $+\mathrm{ve}$ & $18.5 \pm 0.45^{\mathrm{d}}$ & $17.0 \pm 0.50^{\mathrm{d}}$ & $15.8 \pm 0.68^{\mathrm{d}}$ & $16.0 \pm 0.52^{\mathrm{d}}$ & $27.6 \pm 0.22^{\mathrm{a}}$ \\
Staphylococcus pyogenes & $+\mathrm{ve}$ & $17.5 \pm 0.40^{\mathrm{d}}$ & $16.4 \pm 0.40^{\mathrm{d}}$ & $\mathrm{NA}$ & $\mathrm{NA}$ & $25.8 \pm 0.14^{\mathrm{a}}$ \\
Bacillus subtilis & $+\mathrm{ve}$ & $18.2 \pm 0.25^{\mathrm{d}}$ & $16.9 \pm 0.44^{\mathrm{d}}$ & $16.0 \pm 0.49^{\mathrm{d}}$ & $17.6 \pm 0.40^{\mathrm{d}}$ & $28.2 \pm 0.33^{\mathrm{a}}$ \\
\hline
\end{tabular}

All data represented as Mean \pm SD; NA: No Activity; Means followed by the same letters aren't statistically significant $(\mathrm{P}>0.05)$.
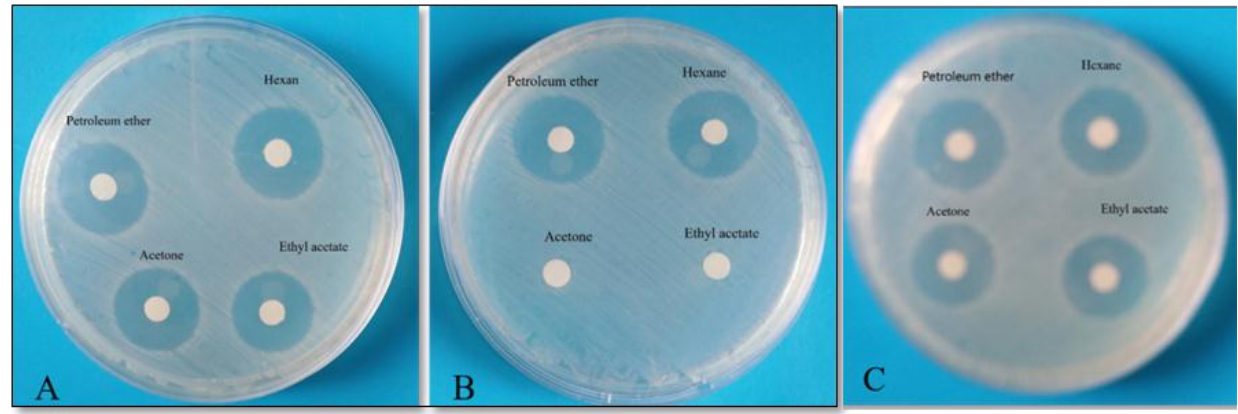

Fig. 1: Antibacterial activity indicated by growth-inhibition zone of Chrysomya albiceps maggots' different crude extracts against Gram-positive bacteria. (A: Staphylococcus aureus; B: Staphylococcus pyogenes; C: Bacillus subtilis)

Results show that, the highest antibacterial activity was recorded by petroleum ether against $S$. aureus with growth-inhibition zone of $(18.5 \pm 0.45 \mathrm{~mm})$, vs. $(27.6 \pm 0.22 \mathrm{~mm})$ for the standard antibiotic (Ampicillin). Meanwhile, petroleum ether and hexane extracts recorded antibacterial activity against $S$. pyogenes with growthinhibition zones of $(17.5 \pm 0.40 \mathrm{~mm}$ and $16.4 \pm 0.40 \mathrm{~mm})$; respectively, compared with $(25.8 \pm 0.14 \mathrm{~mm})$ for the standard antibiotic (Ampicillin).

Table 2: Antibacterial activity of Chrysomya albiceps maggots' extracts against different strains of Gram-negative bacteria.

\begin{tabular}{|c|c|c|c|c|c|c|}
\hline \multirow[t]{2}{*}{ Bacteria } & \multirow{2}{*}{$\begin{array}{c}\text { Gram } \\
+/-\end{array}$} & \multicolumn{4}{|c|}{ Growth-inhibition zone in $\mathrm{mm}$ caused by extracts } & \multirow{2}{*}{$\begin{array}{c}\text { Standard } \\
(\text { Gentamycin })\end{array}$} \\
\hline & & $\begin{array}{c}\text { Petroleum } \\
\text { ether }\end{array}$ & Hexane & Acetone & $\begin{array}{c}\text { Ethyl } \\
\text { acetate }\end{array}$ & \\
\hline Escherichia coli & $-\mathrm{ve}$ & $17.3 \pm 0.40^{\mathrm{d}}$ & $16.8 \pm 0.40^{\mathrm{d}}$ & NA & $16.5 \pm 0.50^{\mathrm{d}}$ & $27.6 \pm 0.10^{\mathrm{a}}$ \\
\hline Klebsiella pneumoniae & $-\mathrm{ve}$ & $16.8 \pm 0.25^{\mathrm{d}}$ & $16.0 \pm 0.57^{\mathrm{d}}$ & NA & NA & $25.2 \pm 0.12^{\mathrm{a}}$ \\
\hline $\begin{array}{l}\text { Pseudomonas aeruginosa } \\
\text { See foot note of Table } 1\end{array}$ & -ve & NA & NA & NA & NA & $22.3 \pm 0.16^{\mathrm{a}}$ \\
\hline
\end{tabular}
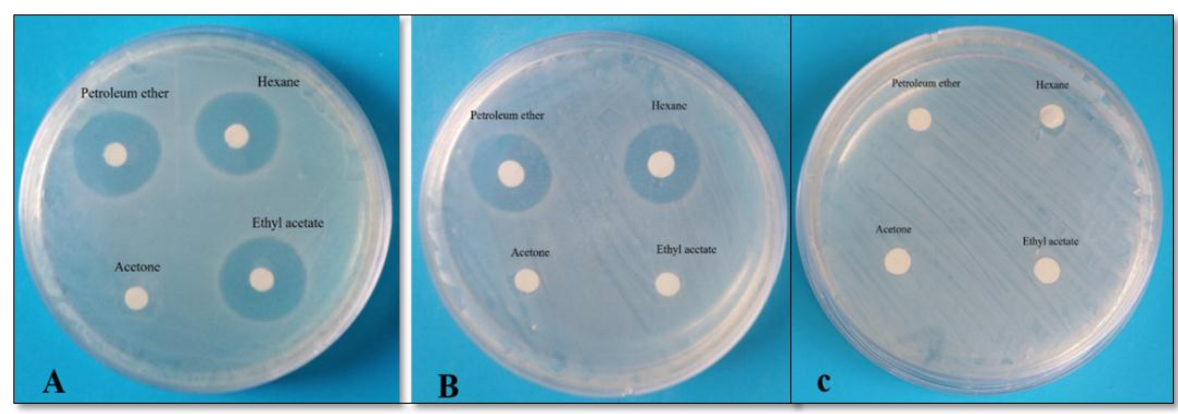

Fig. 2: Antibacterial activity indicated by growth-inhibition zone of Chrysomya albiceps maggots' different crude extracts against Gram-negative bacteria. (A: Escherichia coli; B: Klebsiella pneumoniae; C: Pseudomonas aeruginosa). 
On the other hand, data given in Table (2) and illustrated in Figures (2A-2C) show that petroleum ether, hexane, and ethyl acetate extracts induced potent antibacterial activity against $E$. coli with growth-inhibition zones of $(17.3 \pm 0.40$, $16.8 \pm 0.40$ and $16.5 \pm 0.50 \mathrm{~mm})$; respectively, compared with $(27.6 \pm 0.10 \mathrm{~mm})$ for the standard antibiotic (Gentamycin). In addition, petroleum ether and hexane extracts exhibited antibacterial activity against $k$. pneumoniae equal to $(16.8 \pm 0.25$ and $16.0 \pm 0.57 \mathrm{~mm})$; respectively, compared with $(25.2 \pm 0.12 \mathrm{~mm})$. All tested extracts recorded no activity against $P$. aeruginosa.

\section{Antifungal activity}

Regarding the antifungal activity, data given in Table (3) and illustrated in Figures (3A-3E) exhibit that petroleum ether, hexane, acetone extracts of $C$. albiceps maggots' whole body showed growth-inhibitory effect against A. flavus and $G$. candidum $(17.6 \pm 0.46,17.0 \pm 0.58,16.4 \pm 0.60$ and $19.7 \pm 0.43,18.0 \pm 0.70,14.6 \pm 0.51$ $\mathrm{mm})$; respectively, compared with (Amphotericin B).

Table 3: Antifungal activity of Chrysomya albiceps maggots' extracts against different strains of fungi.

\begin{tabular}{|c|c|c|c|c|c|}
\hline \multirow[b]{2}{*}{ Fungi } & \multicolumn{4}{|c|}{ Growth-inhibition zone in mm caused by extracts } & \multirow{2}{*}{$\begin{array}{c}\text { Standard } \\
\text { (Amphotericin } \\
\text { B) }\end{array}$} \\
\hline & $\begin{array}{c}\text { Petroleum } \\
\text { ether }\end{array}$ & Hexane & Acetone & Ethyl acetate & \\
\hline Aspergillus flavus & $17.6 \pm 0.46^{\mathrm{d}}$ & $17.0 \pm 0.58^{\mathrm{d}}$ & $16.4 \pm 0.60^{\mathrm{d}}$ & NA & $24.6 \pm 0.29^{\mathrm{a}}$ \\
\hline $\begin{array}{l}\text { Aspergillus } \\
\text { fumigatus }\end{array}$ & $16.5 \pm 0.44^{\mathrm{d}}$ & $15.8 \pm 0.52^{\mathrm{d}}$ & NA & $15.0 \pm 0.44^{\mathrm{d}}$ & $25.8 \pm 0.17^{\mathrm{a}}$ \\
\hline Candida albicans & $17.7 \pm 0.73^{\mathrm{d}}$ & $16.8 \pm 0.36^{\mathrm{d}}$ & NA & NA & $21.6 \pm 0.14^{\mathrm{a}}$ \\
\hline Geotricum candidum & $19.7 \pm 0.43^{\mathrm{d}}$ & $18.0 \pm 0.70^{\mathrm{d}}$ & $14.6 \pm 0.51^{\mathrm{d}}$ & NA & $23.0 \pm 0.10^{\mathrm{a}}$ \\
\hline Penicillium sp. & NA & NA & NA & NA & $24.0 \pm 0.20^{\mathrm{a}}$ \\
\hline
\end{tabular}

See foot note of Table 1

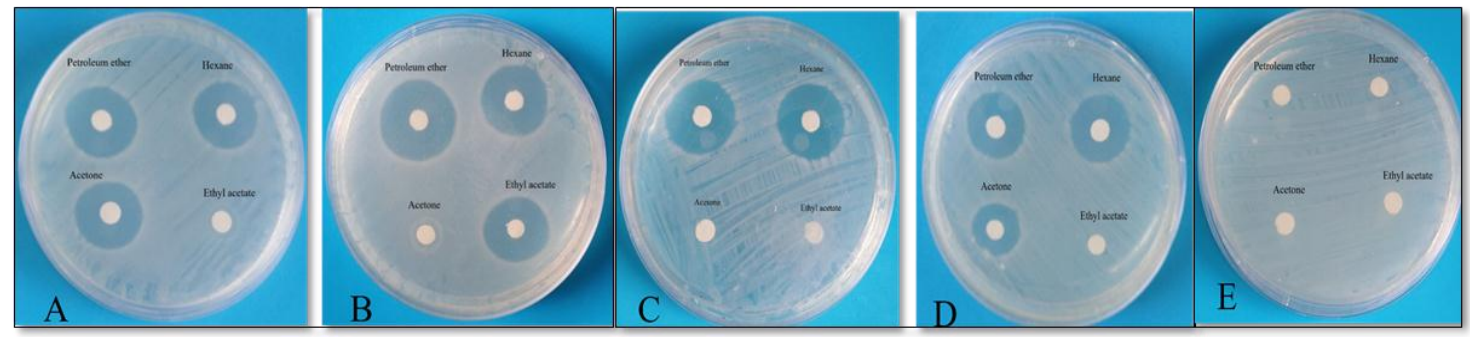

Fig. 3: Antifungal activity indicated by growth-inhibition zone of Chrysomya albiceps maggots' different crude extracts against fungi strains. (A: Aspergillus flavus; B: Aspergillus fumigatus; C: Candida albicans; D: Geotricum candidum; E: Penicillium sp.).

Whereas, petroleum ether, hexane and ethyl acetate extracts recorded growthinhibition zones of $(16.5 \pm 0.44,15.8 \pm 0.52$ and $15.0 \pm 0.44 \mathrm{~mm})$ for A. fumigatus, compared with $25.8 \pm 0.17 \mathrm{~mm}$ for the standard (Amphotericin B). All tested extracts showed no activity against Penicillium sp. Generally, petroleum ether extract showed much activity against all fungal strains tested than those of hexane, acetone and ethyl acetate.

Determination of Minimum Inhibitory Concentration (MIC) by Microdilution Method

The antibacterial activity of $C$. albiceps maggots' crude extracts showed that tested extracts induced the growth of Gram-positive bacteria tested except acetone and ethyl acetate extracts against $S$. pyogenes (Table 4). 
Table 4: Antibacterial activity of Chrysomya albiceps maggots' extracts as indicated by Microdilution plate at $480 \mathrm{~nm}$

\begin{tabular}{|c|c|c|c|c|c|c|}
\hline & \multirow[b]{2}{*}{ Bacterial strains } & \multirow{2}{*}{$\begin{array}{l}\text { Conc. } \\
(\mathrm{mg} / \mathrm{ml})\end{array}$} & \multicolumn{4}{|c|}{ Chrysomya albiceps maggots' different extracts } \\
\hline & & & $\begin{array}{l}\text { Petroleum } \\
\text { ether }\end{array}$ & Hexane & Acetone & $\begin{array}{c}\text { Ethyl } \\
\text { acetate }\end{array}$ \\
\hline \multirow[t]{12}{*}{ G+ve } & Staphylococcus aureus & Control & $4.7 \pm 0.3^{\mathrm{a}}$ & $4.4 \pm 0.5^{\mathrm{a}}$ & $4.3 \pm 0.6^{\mathrm{a}}$ & $4.0 \pm 0.3^{\mathrm{a}}$ \\
\hline & & $\mathbf{5 0 . 0}$ & $1.5 \pm 0.1^{\mathrm{d}}$ & $1.7 \pm 0.2^{\mathrm{d}}$ & $1.6 \pm 0.5^{\mathrm{d}}$ & $1.7 \pm 0.5^{\mathrm{d}}$ \\
\hline & & 25.0 & $1.5 \pm 0.4^{\mathrm{d}}$ & $1.5 \pm 0.1^{\mathrm{d}}$ & $1.7 \pm 0.2^{\mathrm{d}}$ & $1.7 \pm 0.4^{\mathrm{d}}$ \\
\hline & & 12.5 & $1.4 \pm 0.2^{\mathrm{d}}$ & $1.5 \pm 0.3^{\mathrm{d}}$ & $1.5 \pm 0.5^{\mathrm{d}}$ & $1.6 \pm 0.2^{\mathrm{d}}$ \\
\hline & Staphylococcus & Control & $4.7 \pm 0.3^{\mathrm{a}}$ & $4.4 \pm 0.5^{\mathrm{a}}$ & $4.3 \pm 0.6^{\mathrm{a}}$ & $4.0 \pm 0.3^{\mathrm{a}}$ \\
\hline & pyogenes & $\mathbf{5 0 . 0}$ & $1.9 \pm 0.1^{\mathrm{d}}$ & $1.9 \pm 0.4^{\mathrm{d}}$ & NA & NA \\
\hline & & 25.0 & $1.9 \pm 0.2^{\mathrm{d}}$ & $1.7 \pm 0.3^{\mathrm{d}}$ & NA & NA \\
\hline & & 12.5 & $1.7 \pm 0.4^{\mathrm{d}}$ & $1.7 \pm 0.4^{\mathrm{d}}$ & NA & NA \\
\hline & Bacillus subtilis & Control & $4.7 \pm 0.3^{\mathrm{a}}$ & $4.4 \pm 0.5^{\mathrm{a}}$ & $4.3 \pm 0.6^{\mathrm{a}}$ & $4.0 \pm 0.3^{\mathrm{a}}$ \\
\hline & & 50.0 & $2.2 \pm 0.6^{\mathrm{d}}$ & $2.2 \pm 0.1^{\mathrm{d}}$ & $2.3 \pm 0.1^{\mathrm{c}}$ & $2.5 \pm 0.1^{\mathrm{d}}$ \\
\hline & & 25.0 & $2.2 \pm 0.1^{\mathrm{d}}$ & $2.3 \pm 0.2^{\mathrm{d}}$ & $2.3 \pm 0.4^{\mathrm{c}}$ & $2.5 \pm 0.4^{\mathrm{d}}$ \\
\hline & & 12.5 & $2.0 \pm 0.3^{\mathrm{d}}$ & $2.3 \pm 0.5^{\mathrm{d}}$ & $2.2 \pm 0.7^{\mathrm{c}}$ & $2.3 \pm 0.1^{\mathrm{d}}$ \\
\hline \multirow[t]{12}{*}{ G-ve } & Escherichia coli & Control & $4.1 \pm 0.7^{\mathrm{a}}$ & $3.9 \pm 0.5^{\mathrm{a}}$ & $3.7 \pm 0.6^{\mathrm{a}}$ & $4.2 \pm 0.4^{\mathrm{a}}$ \\
\hline & & $\mathbf{5 0 . 0}$ & $2.4 \pm 0.1^{\mathrm{d}}$ & $2.4 \pm 0.2^{\mathrm{d}}$ & NA & $2.5 \pm 0.1^{\mathrm{d}}$ \\
\hline & & 25.0 & $2.2 \pm 0.2^{\mathrm{d}}$ & $2.4 \pm 0.1^{\mathrm{d}}$ & NA & $2.3 \pm 0.4^{\mathrm{d}}$ \\
\hline & & 12.5 & $2.2 \pm 0.2^{\mathrm{d}}$ & $2.3 \pm 0.4^{\mathrm{d}}$ & NA & $2.4 \pm 0.2^{\mathrm{d}}$ \\
\hline & Klebsiella pneumoniae & Control & $4.1 \pm 0.7^{\mathrm{a}}$ & $3.9 \pm 0.5^{\mathrm{a}}$ & $3.7 \pm 0.6^{\mathrm{a}}$ & $4.2 \pm 0.4^{\mathrm{a}}$ \\
\hline & & $\mathbf{5 0 . 0}$ & $2.5 \pm 0.4^{\mathrm{d}}$ & $2.6 \pm 0.2^{\mathrm{d}}$ & NA & NA \\
\hline & & 25.0 & $2.4 \pm 0.4^{\mathrm{d}}$ & $2.5 \pm 0.1^{\mathrm{d}}$ & NA & NA \\
\hline & & 12.5 & $2.3 \pm 0.3^{\mathrm{d}}$ & $2.5 \pm 0.3^{\mathrm{d}}$ & NA & NA \\
\hline & Pseudomonas & Control & $4.1 \pm 0.7^{\mathrm{a}}$ & $3.9 \pm 0.5^{\mathrm{a}}$ & $3.7 \pm 0.6^{\mathrm{a}}$ & $4.2 \pm 0.4^{\mathrm{a}}$ \\
\hline & aeruginosa & $\mathbf{5 0 . 0}$ & NA & NA & NA & NA \\
\hline & & 25.0 & NA & NA & NA & NA \\
\hline & & 12.5 & NA & NA & NA & NA \\
\hline
\end{tabular}

See foot note of Table 1

The lowest MIC value $(12.5 \mathrm{mg} / \mathrm{ml})$ was recorded with petroleum ether extract against $B$. subtilis. Concerning the antibacterial activity against Gram-negative bacteria, acetone extract recorded no activity at all. Ethyl acetate extract has no activity against $K$. pneumoniae and $P$. aeruginosa. Also, all tested extracts have no activity against $P$. aeruginosa (table 4 ). The lowest MIC value was recorded by petroleum ether extract against $E$. coli at $25.0 \mathrm{mg} / \mathrm{ml}$.

Antiviral Assay

The antiviral activity of $C$. albiceps maggots' petroleum ether extracts against Herpes simplex virus (HSV-1) was tested and the maximum non-toxic concentration (MNTC) was determined. The MNTC of tested $C$. albiceps extract recorded $39.06 \mu \mathrm{g} / \mathrm{ml}$. The obtained results revealed that, the tested extract was effective as anti-HSV (Table 5) and Figure (4).

Table 5: Antiviral activity of tested maggots' petroleum ether extracts against Herpes simplex virus (HSV-1) using methyl thiazolyltetrazolium (MTT) assay protocol.

\begin{tabular}{|c|c|c|c|c|c|c|c|c|c|}
\hline Test & $\begin{array}{l}\text { Conc. } \\
(\mu \mathrm{g} / \mathrm{ml})\end{array}$ & & O.D & & $\begin{array}{c}\text { Mean } \\
\text { O.D }\end{array}$ & Viability & Toxicity & $\begin{array}{c}\text { Viral } \\
\text { activity } \\
\%\end{array}$ & $\begin{array}{c}\text { Anti-viral } \\
\text { effect } \\
\%\end{array}$ \\
\hline Control Vero & -- & 0.272 & 0.295 & 0.282 & 0.283 & 100 & 0 & - & - \\
\hline HSV 1 & -- & 0.112 & 0.124 & 0.123 & 0110667 & 42.28504 & 57.71496 & 100 & 0 \\
\hline C. albiceps & 39.06 & 0.278 & 0.284 & 0.264 & 0.275333 & 97.29093 & 2.709069 & 4.693874 & 95.3061258 \\
\hline M. domestica & 2.44 & 0.154 & 0.152 & 0.166 & 0.157333 & 55.59482 & 44.40518 & 76.93872 & 23.06127945 \\
\hline
\end{tabular}



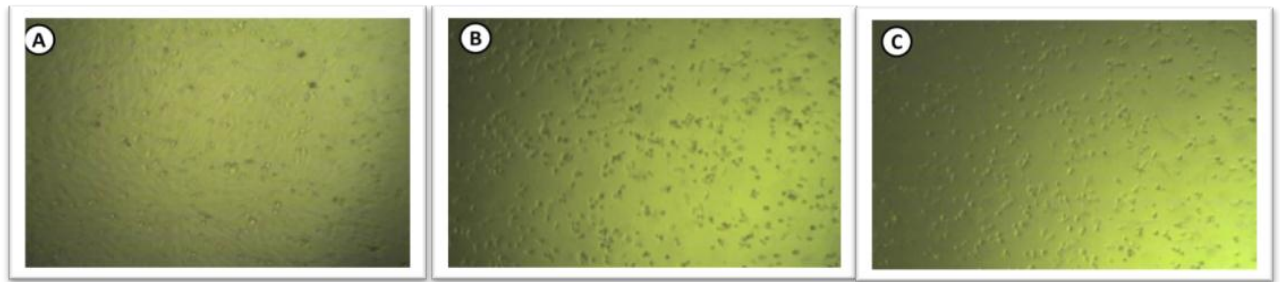

Fig. 4: Activity of maggots' petroleum ether extracts against HSV-1 at $39.06 \mu \mathrm{g} / \mathrm{ml}$. (A) Chrysomya albiceps maggots' extract; (B) M. domestica maggots' extract, and (C) Control.

\section{The house fly, Musca domestica}

\section{Antimicrobial activity using well diffusion method Antibacterial activity}

Antibacterial activity results of $M$. domestica maggots' whole body extracts showed that the highest activity against $S$. aureus was recorded by petroleum ether extract $(13.6 \pm 0.36 \mathrm{~mm})$. Petroleum ether and hexane extracts showed growthinhibition zones of $(14.0 \pm 0.52,15.5 \pm 0.47 \mathrm{~mm})$ against $B$. subtilis; respectively, compared with $(28.2 \pm 0.33 \mathrm{~mm})$ for the standard (Ampicillin), whereas all extracts exhibited no activity against $S$. pyogenes (Table 6 ) and figures $(5 \mathrm{~A}-5 \mathrm{C})$.

Table 6: Antibacterial activity as indicated by growth-inhibition zone of Musca domestica maggots' extracts against different strains of Gram-positive bacteria.

\begin{tabular}{|c|c|c|c|c|c|c|}
\hline \multirow{2}{*}{ Bacteria } & \multirow{2}{*}{$\begin{array}{c}\text { Gram } \\
+/-\end{array}$} & \multicolumn{4}{|c|}{ Growth-inhibition zone in $\mathrm{mm}$ caused by extracts } & \multirow{2}{*}{$\begin{array}{c}\text { Standard } \\
\text { (Ampicillin) }\end{array}$} \\
\hline & & Petroleum ether & Hexane & Acetone & Ethyl acetate & \\
\hline Staphylococcus aureus & $+\mathbf{e e}$ & $13.6 \pm 0.36^{d}$ & $12.2 \pm 0.50^{d}$ & $11.0 \pm 0.22^{d}$ & $12.7 \pm 0.47^{d}$ & $27.6 \pm 0.22^{a}$ \\
\hline Staphylococcus pyogenes & +ve & NA & NA & NA & NA & $25.8 \pm 0.14^{a}$ \\
\hline Bacillus subtilis & +ve & $14.0 \pm 0.52^{\mathrm{d}}$ & $15.5 \pm 0.47^{d}$ & NA & NA & $28.2 \pm 0.33^{a}$ \\
\hline
\end{tabular}

See foot note of Table 1
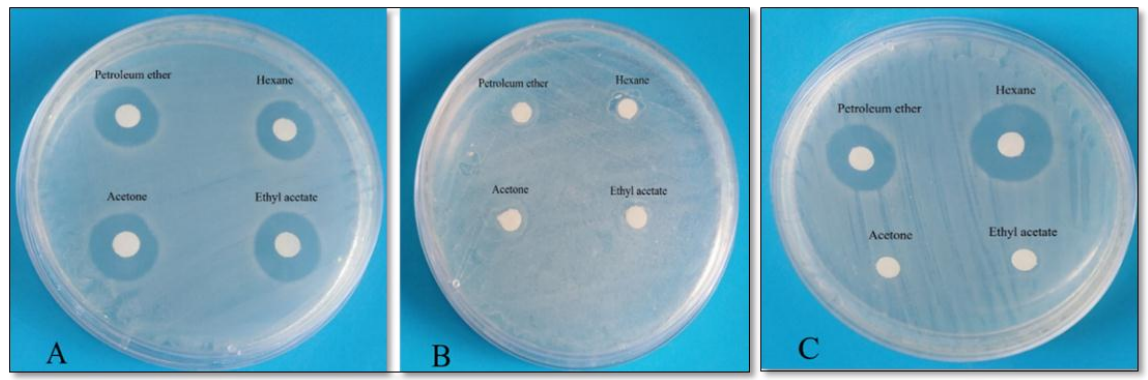

Fig. 5: Antibacterial activity indicated by growth-inhibition zone of Musca domestica maggots' different crude extracts against Gram-positive bacteria. (A: Staphylococcus aureus; B: Staphylococcus pyogenes; C: Bacillus subtilis).

On the other hand, tested extracts showed antibacterial activity against $E$. coli equal to $(14.3 \pm 0.57,13.0 \pm 0.51$ and $12.0 \pm 0.36 \mathrm{~mm})$ for petroleum ether, hexane and ethyl acetate extracts; respectively. While, petroleum ether extract recorded activity against $K$. pneumonia with growth-inhibition zone of $(14.8 \pm 0.44 \mathrm{~mm})$, compared with $(25.2 \pm 0.12 \mathrm{~mm})$ for the standard antibiotic (Gentamycin). Tested extracts exhibit no activity against $P$. aeruginosa tested, (Table 7) and figures (6A-6C).

Table 7: Antibacterial activity as indicated by growth-inhibition zone of Musca domestica maggots' extracts against different strains of Gram-negative bacteria.

\begin{tabular}{|c|c|c|c|c|c|c|}
\hline \multirow{2}{*}{ Bacteria } & \multirow{2}{*}{$\begin{array}{c}\text { Gram } \\
+/-\end{array}$} & \multicolumn{4}{|c|}{ Growth-inhibition zone in $\mathrm{mm}$ caused by extracts } & \multirow{2}{*}{$\begin{array}{c}\text { Standard } \\
\text { Ampicillin }\end{array}$} \\
\hline & & Petroleum ether & Hexane & Acetone & Ethyl acetate & \\
\hline Escherichia coli & -ve & $14.3 \pm 0.57^{d}$ & $13.0 \pm 0.51^{d}$ & NA & $12.0 \pm 0.36^{d}$ & $27.6 \pm 0.10^{\mathrm{a}}$ \\
\hline Klebsiella pneumoniae & -ve & $14.8 \pm 0.44^{\mathrm{d}}$ & NA & NA & NA & $25.2 \pm 0.12^{\mathrm{a}}$ \\
\hline Pseudomonas aeruginosa & -ve & NA & NA & NA & NA & $22.3 \pm 0.16^{\mathrm{a}}$ \\
\hline
\end{tabular}

See foot note of Table 1 


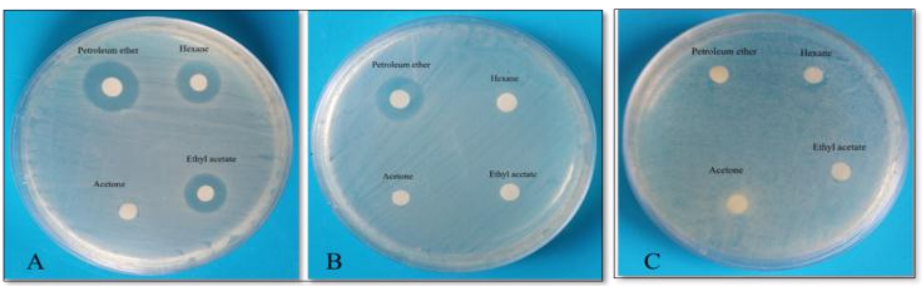

Fig. 6: Antibacterial activity indicated by growth-inhibition zone of Musca domestica maggots' different crude extracts against Gram-negative bacteria. (A: Escherichia coli; B: Klebsiella pneumoniae; C: Pseudomonas aeruginosa).

\section{Antifungal activity}

As shown from the results given in Table (8) and illustrated in Figures (7A-7E), both petroleum ether and hexane extracts of $M$. domestica maggots' exhibited growthinhibition zones against $A$. flavus of $(12.7 \pm 0.24$, and $12.0 \pm 0.58 \mathrm{~mm})$ respectively, compared with $(24.6 \pm 0.29 \mathrm{~mm})$ for the standard (Amphotericin B).

Table 8: Antifungal activity as indicated by growth-inhibition zone of Musca domestica maggots' extracts against different strains of fungi.

\begin{tabular}{|c|c|c|c|c|c|}
\hline \multirow{2}{*}{ Fungi } & \multicolumn{4}{|c|}{ Growth-inhibition zone in mm caused by extracts } & \multirow{2}{*}{$\begin{array}{c}\text { Standard } \\
(\text { Amphotericin B })\end{array}$} \\
\hline & Petroleum ether & Hexane & Acetone & Ethyl acetate & \\
\hline Aspergillus flavus & $12.7 \pm 0.24^{\mathrm{d}}$ & $12.0 \pm 0.58^{d}$ & NA & NA & $24.6 \pm 0.29^{a}$ \\
\hline Aspergillus fumigatus & $11.4 \pm 0.36^{\mathrm{d}}$ & $10.7 \pm 0.70^{d}$ & $9.4 \pm 0.34^{\mathrm{d}}$ & NA & $25.8 \pm 0.17^{\mathrm{a}}$ \\
\hline Candida albicans & $12.1 \pm 0.35^{\mathrm{d}}$ & NA & NA & NA & $21.6 \pm 0.14^{a}$ \\
\hline Geotricum candidum & $13.2 \pm 0.50^{d}$ & $12 . \pm 0.40^{d}$ & NA & NA & $23.0 \pm 0.10^{\mathrm{a}}$ \\
\hline Penicillium sp. & NA & NA & NA & NA & $24.0 \pm 0.20^{a}$ \\
\hline
\end{tabular}

See foot note of Table 1
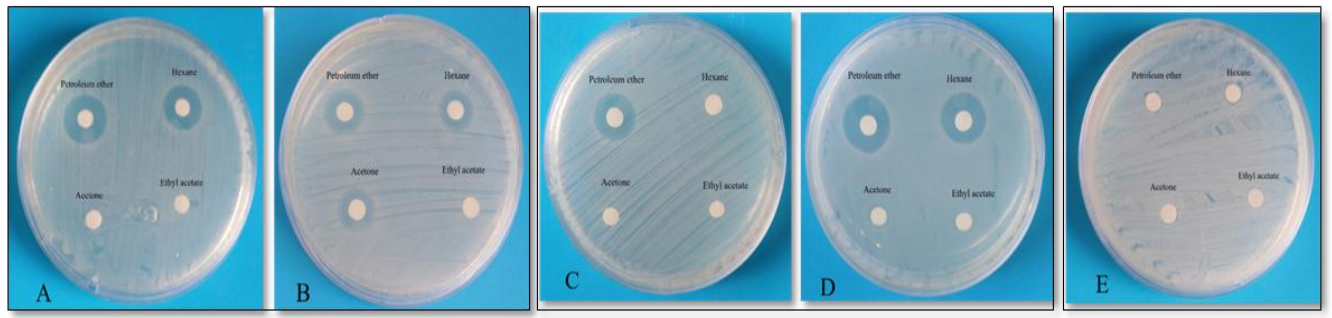

Fig. 7: Antifungal activity indicated by growth-inhibition zone of Musca domestica maggots' different crude extracts against fungi strains. (A: Aspergillus flavus; B: Aspergillus fumigatus; C: Candida albicans; D: Geotricum candidum; E: Penicillium sp.).

The highest growth-inhibition zone against A. fumigatus $(11.4 \pm 0.36 \mathrm{~mm})$ was recorded by petroleum ether. On the other hand, ethyl acetate extract didn't show any activity against all tested fungi species.

\section{Determination of Minimum Inhibitory Concentration (MIC) by Microdilution Method}

None of the tested extracts recorded antibacterial activity against S. pyogenes. Acetone and ethyl acetate extracts of $M$. domestica maggots' showed no activity against $B$. subtilis. The lowest MIC value $50.0 \mathrm{mg} / \mathrm{ml}$ was recorded by petroleum ether, hexane, and ethyl acetate extracts against $S$. aureus, while the same value was obtained for petroleum ether and hexane extracts against $B$. subtilis. On the other hand, petroleum ether extract showed activity against $E$. coli and $K$. pneumoniae, while all tested extracts recorded no activity against $P$. aeruginosa. The lowest MIC value $50.0 \mathrm{mg} / \mathrm{ml}$ was recorded by petroleum ether and hexane against $E$. coli and petroleum ether against $K$. pneumoniae (Table 9). 
Table 9: Antibacterial activity of Musca domestica maggots' extracts as indicated by Microdilution plate at $480 \mathrm{~nm}$

\begin{tabular}{|c|c|c|c|c|c|c|}
\hline \multirow{2}{*}{\multicolumn{2}{|c|}{ Bacterial strains }} & \multirow{2}{*}{$\begin{array}{c}\text { Conc. } \\
(\mathrm{mg} / \mathrm{ml})\end{array}$} & \multicolumn{4}{|c|}{ Musca domestica maggots' different extracts } \\
\hline & & & Petroleum ether & Hexane & Acetone & Ethyl acetate \\
\hline \multirow[t]{12}{*}{ G+ve } & Staphylococcus aureus & Control & $4.7 \pm 0.3^{\mathrm{a}}$ & $4.4 \pm 0.5^{\mathrm{a}}$ & $4.3 \pm 0.6^{\mathrm{a}}$ & $4.0 \pm 0.3^{\mathrm{a}}$ \\
\hline & & $\mathbf{5 0 . 0}$ & $3.2 \pm 0.1^{\mathrm{d}}$ & $3.2 \pm 0.4^{\mathrm{b}}$ & $3.3 \pm 0.2^{\mathrm{a}}$ & $3.3 \pm 0.5^{\mathrm{a}}$ \\
\hline & & 25.0 & $3.2 \pm 0.2^{\mathrm{d}}$ & $3.1 \pm 0.1^{\mathrm{b}}$ & $3.2 \pm 0.6^{\mathrm{a}}$ & $3.3 \pm 0.2^{\mathrm{a}}$ \\
\hline & & 12.5 & $2.9 \pm 0.2^{\mathrm{d}}$ & $3.1 \pm 0.4^{\mathrm{b}}$ & $3.0 \pm 0.2^{\mathrm{b}}$ & $3.2 \pm 0.1^{\mathrm{a}}$ \\
\hline & Staphylococcus pyogenes & Control & $4.7 \pm 0.3^{\mathrm{a}}$ & $4.4 \pm 0.5^{\mathrm{a}}$ & $4.3 \pm 0.6^{\mathrm{a}}$ & $4.0 \pm 0.3^{\mathrm{a}}$ \\
\hline & & 50.0 & NA & NA & NA & NA \\
\hline & & 25.0 & NA & NA & NA & NA \\
\hline & & 12.5 & NA & NA & NA & NA \\
\hline & Bacillus subtilis & Control & $4.7 \pm 0.3^{\mathrm{a}}$ & $4.4 \pm 0.5^{\mathrm{a}}$ & $4.3 \pm 0.6^{\mathrm{a}}$ & $4.0 \pm 0.3^{\mathrm{a}}$ \\
\hline & & 50.0 & $3.2 \pm 0.1^{\mathrm{c}}$ & $3.4 \pm 0.2^{\mathrm{b}}$ & NA & NA \\
\hline & & 25.0 & $3.2 \pm 0.6^{\mathrm{c}}$ & $3.1 \pm 0.1^{\mathrm{b}}$ & NA & NA \\
\hline & & 12.5 & $3.1 \pm 0.2^{c}$ & $3.1 \pm 0.5^{b}$ & NA & NA \\
\hline \multirow[t]{12}{*}{ G-ve } & Escherichia coli & Control & $4.1 \pm 0.7^{\mathrm{a}}$ & $3.9 \pm 0.5^{\mathrm{a}}$ & $3.7 \pm 0.6^{\mathrm{a}}$ & $4.2 \pm 0.4^{\mathrm{a}}$ \\
\hline & & $\mathbf{5 0 . 0}$ & $3.3 \pm 0.4^{\mathrm{b}}$ & $3.2 \pm 0.2^{\mathrm{c}}$ & NA & $3.6 \pm 0.1^{\mathrm{a}}$ \\
\hline & & 25.0 & $3.3 \pm 0.6^{\mathrm{b}}$ & $3.4 \pm 0.1^{\mathrm{c}}$ & NA & $3.4 \pm 0.3^{b}$ \\
\hline & & 12.5 & $3.2 \pm 0.4^{\mathrm{b}}$ & $3.2 \pm 0.1^{\mathrm{c}}$ & NA & $3.4 \pm 0.1^{\mathrm{b}}$ \\
\hline & Klebsiella pneumoniae & Control & $4.1 \pm 0.7^{\mathrm{a}}$ & $3.9 \pm 0.5^{\mathrm{a}}$ & $3.7 \pm 0.6^{\mathrm{a}}$ & $4.2 \pm 0.4^{\mathrm{a}}$ \\
\hline & & $\mathbf{5 0 . 0}$ & $3.5 \pm 0.1^{\mathrm{b}}$ & NA & NA & NA \\
\hline & & 25.0 & $3.4 \pm 0.4^{\mathrm{b}}$ & NA & NA & NA \\
\hline & & 12.5 & $3.4 \pm 0.2^{\mathrm{b}}$ & NA & NA & NA \\
\hline & Pseudomonas aeruginosa & Control & $4.1 \pm 0.7^{\mathrm{a}}$ & $3.9 \pm 0.5^{\mathrm{a}}$ & $3.7 \pm 0.6^{\mathrm{a}}$ & $4.2 \pm 0.4^{\mathrm{a}}$ \\
\hline & & $\mathbf{5 0 . 0}$ & NA & NA & NA & NA \\
\hline & & 25.0 & NA & NA & NA & NA \\
\hline & & 12.5 & NA & NA & NA & NA \\
\hline
\end{tabular}

See foot note of Table 1

\section{Antiviral Assay}

The antiviral activity of $M$. domestica maggots' petroleum ether extracts against Herpes simplex virus (HSV-1) was tested and the maximum non-toxic concentration (MNTC) was determined. The MNTC of tested $M$. domestica extract recorded $2.44 \mu \mathrm{g} / \mathrm{ml}$. The obtained results revealed that, the tested extract was effective as antiHSV (Table 5 and Fig. 4).

\section{DISCUSSION}

There is already a long history of use of insects in Folk Medicine (Ratcliffe et al.2014). The use of insects in Folk Medicine encourage the scientists to develop potential new medicines for treating serious diseases such as viral infections and problems associated with the newly emerging antibiotic-resistance. The present study aimed at evaluate the antimicrobial activity of $C$. albiceps and $M$. domestica maggots' whole body extracts using Well diffusion and Microdilution methods, and to determine the activity of these maggots' petroleum ether extract as anti-HSV-1.

Insects are known to possess well developed immune system that forms a potent defense against any invading bacteria (Gotz and Boman, 1985; kimbrell, 1991; Hasaballah 2018). In cellular immunity, mechanisms such as phagocytosis and encapsulation are operative (Boman and Hultmark, 1987), while humoral responses mainly involve the production of a variety of antibacterial and antifungal proteins that are induced or increased in response to infection (Abraham et al. 1995).

In this study, the antibacterial activity results showed that, tested extracts evoked a variable activity against both Gram-positive bacteria and Gram-negative bacteria depending on the solvent used in extraction. Generally, petroleum ether was the most effective against different bacteria species followed by hexane, ethyl acetate and acetone extract. Also, Gram-positive bacterial strains were more sensitive to the tested maggots' extracts than Gram-negative bacterial strains. Similar results were 
observed by Leem et al. (1999) using, Acantholyda parki isolates as a broad antibacterial spectrum against both Gram-negative and Gram-positive bacteria.

Insects body produce combinations of antibacterial peptides in response to natural infection leading to a broad spectrum activity against micro-organisms (Yamauchi, 2001). In spite of such a response, the susceptible insects within the host range of a given pathogen are successfully killed by the pathogen and in contrast, the insects resistant against the pathogen appear to be out of the host range.

In this study, the antibacterial activity of $C$. albiceps maggots' extract was more effective than those of $M$. domestica, in the same context Thomas et al. (1999), concluded that antibacterial effect of different extracts was arranged as the following: Lucilia sericata maggots' extracts was more effective than $C$. albiceps; $S$. carnaria and $M$. domestica, where these extracts were able to decrease the total bacterial count of $S$. aureus in vitro and to combat clinical infections in a variety of wound types including these caused by antibiotic-resistant strains. Such findings may be due to the presence of antibacterial agents in either their body or their secretion/excretion (Amer et al. 2019).

On the other hand, tested extracts showed a variable antifungal activity against A. flavus, A. fumigatus, C. albicans and G. candidum fungal strains with no activity against Penicillium. In general, petroleum ether was more effective than those of hexane, ethyl acetate and acetone. However, the present study has shown that the bacterial strains tested were more sensitive to tested extracts used than the fungal strains. In agreement with these results, Meylaers et al. (2004) recorded antibacterial activity of $M$. domestica methanolic whole body extract against Saccharomyces cerevisiae. Hou et al. (2007) stated that, the housefly larvae have higher activity against Gram-positive bacteria than Gram-negative bacteria without any antifungal activity. Similar results was also recorded by Yamada and Natori, (1994) with, $S$. peregrine; Rees et al .(1997) using, B. pascuorum, Leem et al. (1999) using, A. parki; Vizioli et al. (2001) using the mosquito vector, Anpheles gambiae; Cytrynska et al. (2007) using, Galleria mellonella.

Petroleum ether of tested extracts showed promising activities as antiHSV-1. In agreement, Esser et al. (1979) treated murine virus capsid with melittin; Wachinger et al. (1998) reduced the viral infectivity with an intracellular action of the peptide taken up into the cells; Baier et al.(2000) who found that, nasal application of lipopeptide increased protection against the lethal infection of influenza; Fenard et al.., (2001) who found that, the honeybee venom inhibits the replication of T-lymphotropic HIV1 isolates; Chernysh et al. (2002) who tested the activity of alloferon against HSV-1 and Hepatitis B\&C infection; Slocinska et al. (2008) who stated that, peptides extracted from insects able to cause antiviral action against HSV; Ai et al. (2008) who stated that, protein fractions extracted and purified from the housefly larvae possess antiviral activity. Moreover, Amer et al.(2019) reported that, insects extracts possess antiviral activity against Hepatitis A Virus (HAV).

\section{CONCLUSION}

From the results demonstrated previously it could be concluded that, $C$. albiceps and $M$. domestica tested maggots' extracts evoked a variable activity against both Gram-positive bacteria and Gram-negative bacteria depending on the solvent used in extraction. Petroleum ether was the most effective against different bacteria species followed by hexane, ethyl acetate and acetone extract. Gram-positive bacterial strains were more sensitive than Gram-negative bacterial strains. Tested extracts also showed a variable antifungal activity. In addition, petroleum ether 
extract may play a role as a potential anti-HSV agent due to their promising antiviral activity.

\section{REFERENCES}

Abraham, E.G.; Nagaraju, J.; Salunke, D.; Gupta, H.M. and Datta, R.K. (1995). Purification and partial characterization of an induced antibacterial protein in the silk worm, Bombyx mori. J. Invert. Path. 65(1): 17-24.

Ahn, M.Y.; Ryu, K.S.; Lee, Y.W. and Kim, Y.S. (2000). Cytotoxicity and L-Amino Acid Oxidase Activity of crude insect drugs. Arch. Pharm. Res. 23(5): 477-481.

Ai, H.; Wang, F.; Yang, Q.; Zhu, F. and Lei, C. (2008). Preparation and biological activities of chitosan from the larvae of housefly, Musca domestica. Carbohydrate Polymers. 72: 419-423.

Amer, M.S.; Hammad, K.M.; Shehata, A.Z.; Hasaballah, A.I. and Zidan, M.M. (2019). Electrophoretic Protein and Amino Acid Analysis of Lucilia sericata, Chrysomya albiceps (Diptera: Calliphoridae) and Musca domestica (Diptera: Muscidae) Larval Excretion/Secretion. Egypt. Acad. J. Biolog. Sci., 11(1): 117- 130.

Armitage, P. (1974). Paired student t-test in statistical methods in medical research black well scientific pub. Oxford, London. pp: 116-120.

Baier, W.; Masihi, N.; Huber, M.; Hoffmann, P. and Bessler, W.G. (2000): Lipopeptides as immune adjuvants and immune stimulants in mucosal immunization. J. Immun. biol., 201(3-4): 391-405.

Boman, H.G. (2003). Antibacterial peptides: basic facts and emerging concepts. J. Int. Med. 254: 197-215.

Boman, H.G. and Hultmark, D. (1987). Cell-free Immunity in Insects. Annu. Rev. Microbiol. 41:103-126.

Bulet, P.; Stocklin, R. and Menin, L. (2004). Anti-microbial peptides: from invertebrates to vertebrates. Immunol. Rev. 198: 169- 184.

Busvine, J.R. (1962). A laboratory technique for measuring the susceptibility of house flies and blow flies to insecticides. Lab. Port., 11: 464-465.

Chernysh, S.; Kim, S.I.; Bekker, G.; Pleskach, V.A.; Filatova, N.A.; Anikin, V.B.; Platonov, V.G. and Bulet, P. (2002). Antiviral and antitumor peptides from insects. JABS. 99(20): 12628-32.

Cociancich, S.; Dupont, A.; Hegy, G.; Lanot, R.; Holder, F.; Hetru, C.; Hoffman, J.A. and Bulet, P. (1994). Novel inducible antibacterial peptides from a hemipteran insect, the sap-sucking bug Pyrrhocoris apterus. Biochem. J. 300: 567- 575.

Cytrynska, M.; Mak, P.; Zdybicka-Barabas, A.; Suder, P. and Jakubowicz, T. (2007). Purification and characterization of eight peptides from Galleria mellonela immune hemolymph. Peptides. 28: 533-546.

Esser, A.F.; Bartholomew, R.M; Jensen, F.C. and Muiler-Eberhard, H.J. (1979). Disassembly of viral membranes by complement independent of channel formation. Proc. Nat. acad. Sci. USA., 76:5843-47.

Fehlbaum, P.; Bulet P.; Michaut L.; Lagueux, M.; Broekaert, W.F.; Hoffmann, J.A. (1994). Insect immunity: septic injury of Drosophila induces the synthesis of a potent antifungal peptide with sequence homology to plant antifungal peptides. J. Biol. Chem. 269: 33159-63.

Fenard, D.; Lambeau, G.; Maurin, T.; Lefebvre, J.C. and Doglio, A. (2001). A peptide derived from bee venom-secreted phospholipase A2 inhibits replication of T-cell tropic HIV-1 strains via interaction with the CXCR4 chemokine receptor. Mol. Pharmacol. 60: 341-347. 
Fouda, M.A.; Hassan, M.I.; Hammad, K.M. and Hasaballah, A.I. (2013). The Effects of Midgut Bacteria and Protease Inhibitors on the Reproductive Potential and Midgut Enzymes of Culex pipiens, Mosquito Infected with Wuchereria Bancrofti Filaria. J. Egypt. Soc. Parasitol. 43(2): 537-545. DOI:10.12816/0006410.

Gotz, P. and Boman, H.G. (1985). Insect immunity in Comprehensive Insect Physiology, Biochemistry and Phamacology (G.A. Kerkut and L.I. Gilbert, Eds.). Perg, Oxford. 453-485.

Hancock, R.E.W. and Rozek, A. (2002). Role of membranes in the activities of antimicrobial cationic peptides. FEMS Microbiol. Lett. 206: 143-149.

Hasaballah, A.I. and Elnaggar, H. (2017): Antimicrobial effects of some Marine Sponges and its Biological and Repellent Activity against Culex pipiens (Diptera: Culicidae). Ann. Res. Rev. Biol. 12(3): 1-14. DOI:10.9734/ARRB/2017/32450.

Hasaballah, A.I., (2015). Toxicity of some plant extracts against vector of lymphatic filariasis, Culex pipiens. J. Egypt. Soc. Parasitol. (JESP). 45(1): 185-193. DOI:10.12816/0010864.

Hasaballah, AI, (2018). Impact of gamma irradiation on the development and reproduction of Culex pipiens (Diptera; Culicidae). Int. J. Rad. Biol. 94(9): 844849. DOI: 10.1080/09553002.2018.1490040.

Hassan, M.I.; Fouda, M.A.; Hammad, K.M. and Hasaballah, A.I. (2013). Effects of midgut bacteria and two protease inhibitors on the transmission of Wuchereria bancrofti by the mosquito vector, Culex pipiens. J. Egypt. Soc. Parasitol. 43(2): 553-559. DOI:10.12816/0006411.

Hoffmann, J.A. (1995). Innate immunity of insects. Curr. Opin. Immunol. 7: 4-10.

Hoffmann, J.A.; Reichhart, J.M. and Hetru, C. (1996): Innate immunity in higher insects. Curr. Opin. Immunol. 8: 8-13.

Hou, L.; Shi, Y.; Zhai, P. and Le, G. (2007). Antibacterial activity and in vitro antitumor activity of the extract o the larvae of the housefly (Musca domestica) J. Ethnoph. 111: 227-231.

Hultmark, D. (1993): Immune reactions in Drosophila and other insect: A model for innate immunity. Tren. Genet., 9: 178-183.

Irith, W.; Kai, H. and Hancock R.E.W. (2008). Agar and broth dilution methods to determine the minimal inhibitory concentration (MIC) of antimicrobial substances. Nature Protocols. 3(2): 163-175.

Januszanis, B.; Staczek, S.; Barabas, A.Z.; Badziul, D.; Gil, J.J.; Langer, E.; Rzeski, W. and Cytrynska, M. (2012). The effect of Galleria mellonella hemolymph polypeptides on human brain glioblastoma multiforme cell line- a preliminary study. Annales UMCS, Biologia. 67 (2): 53- 62.

Kimbrell, D. A. (1991). Insect antibacterial proteins: not just for insects and against bacteria. BioEssays. 13(12): 657-663.

Lamberty, M.; Ades, S.; Uttenweiler-Joseph, S.; Brookhart, G.; Bushey, D.; Hoffmann, J.A. and Bulet, P. (1999). Insect Immunity: Isolation from the lepidopteran Heliothes virescense of a novel insect defensin with potent antifungal activity. J. Biol. Chem. 274(14): 9320-26.

Leem, J.Y.; Jeong, I.J.; Prak, K.T. and Park, H.Y. (1999). Isolation of Phydroxycinnamaldehyde as an antibacterial substance from the saw fly, Acantholyda Parki S. FEBS Lett. 442: 53-56.

Lentner, C.; Lentner, C. and Wink, A. (1982). Students t- distribution tables. In Geigy scientific Tables Vol. 2. International Medical and Pharmaceutical information, Ciba- Geigy Limited, Basal, Switzerland. 
Levashina, E.A.; Ohresser, S.; Bulet, P.; Reichhart, J.M.; Hetru, C. and Hoffmann, J.A. (1995). Metchinikowin, a nobel immune-inducible proline- rich peptide from Drosophila with antibacterial and antifungal properties. Eur. J. Biochem. 233: 694-700.

Meylaers, K.; Cerstiaens, A.; Vierstraete, E.; Baggerman, G.; Michiels, C.W.; De Loof, A. and Schoofs, L. (2002). Antimicrobial Compounds of Low Molecular Mass are Constitutively Present in Insects: Characterization of $\beta$-AlanylTyrosine. Curr. Pharm. Design, 8: 99-110.

Meylaers, K.; Clymen, E.; Daloze, D.; Deloof, A. and Schoofs, L. (2004). Identification of 1-lysophos- phatidylethanolamine (C16:1) as an antimicrobial compound in the housefly, Musca domestica. Insect Biochem. Mol. Biol. 34(1): 43-49.

Pemberton, R.W. (1999). Insects and other arthropods used as drugs in Korean traditional medicine. J. Ethnoph, 65: 207-216.

Ratcliffe, N.; Patricia, A.; Cicero, B.M. (2014): Recent advances in developing insect natural products as potential modern-day medicines. J. Evid. Based Com.. Altern. Med.

Rees, J.A.; Moniatte, M. and Bulet, P. (1997). Novel antibacterial peptides isolated from a European Bumblebee Bumbus pascurum (Hymenoptera, Apoidea). Insect Biochem. Mol. Biol. 27(5): 413-422.

Slocinska, M.; Marciniak, P. and Rosinski, G. (2008). Insects Antiviral and Anticancer Peptides: New Leads for the Future? Protein peptide lett. 15. 57885. 10.2174/092986608784966912.

Tadashi, A. (1975). Culture media for actinomycetes. The Society for actinomycetes, Japan National agricultural Library. 1: 1-31.

Thomas, S.; Andrews, A.M.; Hay, N.P. and Bourgoise, S. (1999). The antimicrobial activity of maggot secretions; result of a preliminary study. J. Viabi. 9: 127132.

Valgas, C.; Souza, S. M.D.; Smânia, E. F. and Smânia Jr, A. (2007). Screening methods to determine antibacterial activity of natural products. Braz. J. Microbiol. 38(2): 369-380.

Vizioli, J.; Richman, A.; Joseph, U.S.; Blass, C. and Bulet, P. (2001). The defensin peptide of the malaria vector mosquito Anopheles gambiae: antimicrobial activities and expression in adult mosquitoes. Insect Biochem. Mol. Biol. 31: 241-248.

Wachinger, M.; Kleinschmidt, A.; Winder, D.; von Pechmann, N.; Ludvigsen, A.; Neumann, M.; Holle, R.; Salmons, B.; Erfle, V. and Brack-Werner, R. (1998). Antimicrobial peptides melittin and cecropin inhibit replication of human immunodeficiency virus 1 by suppressing viral gene expression. J. Gen. Virol. 79: 731-740.

Wohlleben, W.; Mast, Y.; Stegmann, E.; and Ziemert, N. (2016). Antibiotic drug discovery. Microb. Biotechnol. 9(5): 541-548.

Yamada, K. and Natori, S. (1994). Characterization of the antimicrobial peptide derived from sapecin B, an antibacterial protein of Sarcophaga peregrina (flesh fly). Biochem. J. 298(3): 623-628.

Yamauchi, H. (2001). Two novel insect defensins from larvae of the cupreous chafer, Anomala cuprea: purification, amino acid sequences and antibacterial activity. Insect Biochem. Mol. Biol. 32: 75-84.

Zasloff, M. (2002). Antimicrobial peptide of multicellular organisms. Nature. 415: 389-395. 